\title{
Utilidad del lavado broncoalveolar en el diagnóstico de la neumonía asociada a la ventilación mecánica
}

\author{
Utility of bronchoalveolar lavage in diagnosing \\ ventilator-associated pneumonia
}

\author{
Guillermo Ortiz, MD. (1); Antonio Lara García, MD. ${ }^{(2)}$; Manuel Garay Fernández, MD. ${ }^{(3)}$
}

\section{Resumen}

La neumonía asociada a la ventilación mecánica es la principal causa de infección y muerte en la unidad de cuidado intensivo. El diagnóstico implica la presencia de signos sistémicos de infección, infiltrados pulmonares nuevos o que empeoran y pruebas bacteriológicas positivas; sin embargo todas estas son características no específicas que pueden ser causadas por múltiples circunstancias en el paciente critico. Además, el inicio tardío o inadecuado de antibióticos también empeora el pronóstico y el desenlace en la neumonía asociada a la ventilación mecánica, por tanto la información microbiológica es de vital importancia para disminuir la brecha del tratamiento empírico.

Las técnicas para toma de muestras invasivas del tracto respiratorio inferior incluyen la fibrobroncoscopia con lavado broncoalveolar o cepillo protegido, cuyas muestras se procesan para obtener el aislamiento cuantitativo de los gérmenes. Las técnicas no broncoscópicas descritas incluyen el aspirado endotraqueal y el mini-lavado broncoalveolar, que tienen un punto de corte diferente en los cultivos para ser considerados positivos. La decisión del mejor método de toma de muestra para el diagnóstico de neumonía asociada a la ventilación mecánica, sigue siendo controversial y hasta ahora ninguno ha demostrado ser superior. Así entonces, se recomienda utilizar aquella técnica que pueda ser obtenida de forma rápida en cada centro por parte de personal experimentado y familiarizado con la misma. La terapia temprana y correcta para el manejo de esta condición ha demostrado su relevancia a través de múltiples estudios que se analizan es este artículo. La interpretación meticulosa de los resultados de los cultivos debe llevar a de-escalar la terapia antibiótica para cumplir con las políticas de uso apropiado de antibióticos y disminución de la mortalidad.

Palabras clave: lavado broncoalveolar, neumonía asociada a la ventilación mecánica, lavado bronquial no broncoscópico.

\footnotetext{
Abstract

Ventilator-associated pneumonia is the main cause of infection and death in the intensive care unit. Its diagnosis implies the presence of systemic signs of infection, new or worsened pulmonary infiltrates, and positive bacteriologic tests; however, these are non-specific characteristics that may be caused by multiple circumstances in the critically ill patient. Moreover, late or inadequate initiation of antibiotic therapy also worsens the prognosis and outcome of ventilator-associated pneumonia, and therefore microbiologic information is of vital importance for reducing the gap of empirical treatment.

One invasive technique for obtaining samples of the lower respiratory tract is bronchoscopy with bronchoalveolar lavage or protected brush. Samples are processed to obtain quantitative isolation of germs. Non-bronchoscopic techniques have also been described, including endotracheal aspiration and mini-bronchoalveolar lavage; the cut-points of these techniques, in order that they may be considered positive, are different. The decision concerning the best method for obtaining samples for diagnosing ventilator-associated pneumonia is still controversial, and until now none of them has proven to be superior. At any rate, it is advisable to use
}

\footnotetext{
(1) Internista, Neumólogo, Intensivista. Jefe Unidad de Cuidado Intensivo. Hospital Santa Clara. Bogotá, Colombia.

${ }^{(2)}$ Internista, Neumólogo, Intensivista. Unidad Cuidado Intensivo. Hospital Santa Clara. Bogotá, Colombia

${ }^{(3)}$ Internista, Neumólogo. Unidad Cuidado Intensivo. Hospital Santa Clara. Bogotá, Colombia.

Correspondencia: Guilleromo Ortiz, correo electrónico: ortiz_guillermo@hotmail. com

Recibido: 10/03/16. Aceptado: 19/03/16.
} 
the technique that may be readily performed in each center by experienced practitioners who are familiar with the technique. Early and adequate therapy for the management of ventilator-associated pneumonia has shown its importance in many studies that are analyzed in this article. Judicious interpretation of culture results should lead to de-escalation of antibiotic therapy, in order to comply with policies concerning adequate antibiotic use and reduction of mortality..

Keywords: bronchoalveolar lavage, ventilator-associated pneumonia, non-bronchoscopic bronchial lavage.

\section{Introducción}

La neumonía asociada a la ventilación mecánica (NAVM), como complicación en pacientes críticamente enfermos, puede ser difícil de distinguir clínicamente de otros procesos patológicos; además, constituye la principal causa de infección y muerte en la unidad de cuidado intensivo $(1,5)$. El diagnóstico de esta entidad se basa en tres componentes: signos sistémicos de infección, opacidades parenquimatosas nuevas o que empeoran en la radiografía de tórax y pruebas bacteriológicas de infección pulmonar $(1,3)$. Para determinar la confiabilidad de estos parámetros se han diseñado diferentes trabajos que demuestran baja confiabilidad del diagnóstico clínico (1). Signos de infección como fiebre, taquicardia y leucocitosis, son inespecíficos y pueden estar causados por cualquier condición que promueva la liberación de citoquinas. Eventos traumáticos, por ejemplo, generan cambios en estos parámetros representando síndrome de respuesta inflamatoria sistémica (SIRS por su sigla en inglés) no infeccioso, que puede llevar a inicio de tratamiento antibiótico no indicado.

El diagnóstico de NAVM requiere, además, cambios en la radiografía de tórax, que suelen ser insuficientes por su bajo grado de especificidad (3, 4). Un estudio observacional demostró que sólo el $43 \%$ de los pacientes que tenían evidencia clínica y radiográfica para el diagnóstico de NAVM, contaron con la confirmación histopatológica mediante autopsia (1), lo que se suma a una alta variabilidad interobservador.

En escenarios aún más complejos, como en lesión pulmonar aguda o síndrome de dificultad respiratoria aguada (SDRA), la aparición de signos de respuesta inflamatoria sistémica y los cambios en la radiología y en la oxigenación, hacen que la predicción clínica de neumonía bacteriana se acerque al 29\% de los casos al compararla con el estudio histopatológico en autopsia (2).

Publicaciones recientes demuestran que el inicio tardío o inadecuado del tratamiento antibiótico incrementa la posibilidad de desenlaces que empeoran el pronóstico clínico e incrementan la mortalidad (6, 7), de ahí que se haga necesario instaurar un cubrimiento antibiótico lo suficientemente amplio para incluir microorganismos propios del ámbito nosocomial (5). Por el contrario, el inicio de antibióticos de espectro excesivo podría determinar alta morbilidad y mortalidad en los pacientes en cuidado crítico (8, 21). De esta manera, la información microbiológica tiene como objetivo ayudar en la disminución del tiempo de tratamiento empírico de amplio espectro tanto como sea posible, garantizado un uso ajustado de los agentes antimicrobianos. Así mismo, aportar en el conocimiento de la microbiología propia del lugar en el cual se lleva a cabo la práctica clínica, son datos que constituyen la base para el inicio empírico de la terapia, teniendo como referencia la ecología propia de la Unidad de Cuidado Intensivo.

\section{Estudio de muestras del tracto respiratorio inferior}

En la aproximación etiológica propuesta en el consenso de la ATS (American Thoracic Society) publicado en el año 2005, los pacientes con sospecha de neumonía asociada al ventilador deben ser sometidos a estudio de muestras del tracto respiratorio inferior, análisis microbiológico y cultivo de las mismas antes del inicio del tratamiento antibiótico (3).

Las técnicas para toma de muestras se dividen en invasivas y no invasivas, de las cuales se obtienen cultivos que pueden ser cuantitativos o cualitativos. 


\section{Técnicas invasivas}

Bajo la utilización de fibrobroncoscopia se obtienen el lavado broncoalveolar (LBA) o el lavado con cepillo protegido (LCP), que al ser procesados permiten el estudio microbiológico en el que se establecen las unidades formadoras de colonia (UFC) del microorganismo implicado, y se identifican puntos de corte específicos para el diagnóstico $\left(10^{4}\right.$ para el LBA y $10^{3} \mathrm{UFC} / \mathrm{ml}$ para el LCP) $(9,28)$, otorgándole características cuantitativas al aislamiento. La sensibilidad reportada para el LCP y el LBA es del 33-100\% y del $42-93 \%$, respectivamente, en tanto que la especificidad es del 50-100\% y del 45-100\% (30). La implementación requiere el uso de solución salina estéril o el dispositivo de cepillo a través del fibrobroncoscopio según técnicas estandarizadas para la realización del procedimiento, respectivamente.

\section{Técnicas no invasivas}

Las técnicas no broncoscópicas incluyen el aspirado endotraqueal (AET) y el mini-BAL, técnicas que requieren la utilización de dispositivos de instilación y succión de solución salina de manera ciega. Algunos trabajos proponen el punto de corte para aspirado endotraqueal (AET) de $10^{6} \mathrm{UFC} / \mathrm{ml}$ con el fin de determinar la significancia del aislamiento (29) con sensibilidad que varía desde 38 hasta $100 \%$ y especificidad de 14 a $100 \%$ (31).

El análisis de muestras de la vía aérea ha incluido desde el Gram de la secreción traqueal hasta cultivos cualitativos y cuantitativos. Del primero, se cuenta en la literatura con diferentes estudios que han planteado su utilidad en el diagnóstico e inicio de la terapia antibiótica. Un metaanálisis (22) reciente publicado por O'Horo et al., evaluó el papel de la tinción de Gram en aislamientos de la vía respiratoria para el diagnóstico de neumonía asociada a la ventilación mecánica (NAVM) frente a cultivo, e informó sensibilidad combinada de tinción de Gram para NAVM de $0,79(0,77-0,8195 \%$ IC, $p<0,0001)$ y especificidad de 0,75 (0,73 a 0,78 IC 95\%, p <0,0001).

El valor predictivo negativo de la tinción de Gram para una prevalencia de $20-30 \%$ de NAVM, es del $91 \%$, es decir que un Gram negativo para bacterias obtenido durante los estudios hace muy poco probable la presencia de una NAVM. Sin embargo, el valor predictivo positivo de la tinción de Gram fue sólo del $40 \%$. El grado de correlación evaluado con el índice Kappa fue de 0,42 para organismos grampositivos y 0,34 para organismos gramnegativos. Por tanto, el resultado de tinción de la grampositiva, no debe utilizarse para establecer la terapia antibiótica hasta que los cultivos estén disponibles.

Tres estudios aleatorizados $(13,15,16)$ publicados por Torres et al., demostraron no encontrar diferencia significativa en morbilidad o mortalidad al comparar técnicas invasivas (LBA/LCP) frente a técnicas no invasivas (AET). No obstante, al evaluar la consistencia de la evidencia, estos ensayos clínicos contaron con un número reducido de pacientes y por consiguiente su poder estadístico puede ser limitado por el tamaño de la muestra, disminuyendo la capacidad de detectar las diferencias entre los grupos analizados.

De otra parte, el estudio de Fagon (12) incluyó 413 pacientes en cuidado intensivo con sospecha clínica de NAVM. Al comparar técnicas invasivas frente a estudios de muestras obtenidas mediante aspirado traqueal, el grupo de diagnóstico con técnica invasiva recibió menos días de antibioticoterapia (11,4 vs. $7,5)$, menor cantidad de antibiótico por día (1,0 vs. 1,3) y menor disfunción orgánica del día 3 al 7. La mortalidad demostrada a los 14 días fue significativamente menor en el grupo de diagnóstico con técnica invasiva ( 16,2 vs. $25,8 \mathrm{p}=0,022)$, sin que se demostrara diferencia en el tiempo evaluado a los 28 días.

Un estudio multicéntrico que contó con 740 pacientes y sospecha de NAVM publicado por el Canadian Critical Care Trial group (19), evaluó la hipótesis, que el cultivo cuantitativo del lavado broncoalveolar (BAL) se asociaba con menores tasas de mortalidad y mayor uso de la terapia antibiótica específica frente a los no cuantitativos al usar aspirado endotraqueal (AET), sin hallar diferencia significativa en términos de mortalidad $(18,9 \%$ y $18,4 \%$, respectivamente; $p=0,94)$, terapia dirigida o días de estancia en UCI o en el hospital. Es importante destacar que los pacientes que se sabían colonizados o infectados por especies de Pseudomonas 
o Staphylococus resistentes a la meticilina se excluyeron del análisis, hecho que generó controversia, dado que la población a la cual se hace frente en cuidado intensivo presenta como principal causa etiológica estos microorganismos (20).

Datos obtenidos en el estudio publicado por Shorr (14) demostraron que el uso de técnicas invasivas podría derivar en cambio en la prescripción, modificando el régimen antibiótico hasta en el 50\% de los pacientes.

La decisión del mejor tipo de muestra para el diagnóstico de NAVM continúa siendo controversial y hasta el momento el tipo de procedimiento para su obtención no ha demostrado ser superior uno al otro $(9,10)$. La muestra recomendada para el diagnóstico es aquella que pueda ser obtenida de forma rápida en cada centro, por el clínico según su grado de experiencia (Guías británicas de diagnóstico y tratamiento de la NAVM) (11).

La terapia temprana y adecuada para el manejo de la NAVM ha demostrado su importancia a través de diferentes estudios, lo cual ha generado un impacto positivo en la mortalidad $(6,17,18)$. En el estudio publicado por Alvares-Lerma (17), hasta el $43 \%$ de los pacientes requirieron modificación del tratamiento antibiótico y la principal causa (62\% de los casos) de cambio fue el cubrimiento inadecuado de microorganismo, con aumento significativo de la mortalidad en dicho grupo. La mortalidad no se ve afectada por el uso o no de la broncoscopia, sino por el inicio adecuado y temprano del tratamiento.

Las técnicas broncoscópicas y no broncoscópicas para el diagnóstico de NAVM han sido comparadas por diferentes autores en un intento por demostrar la utilidad de las mismas (12-14). La evidencia demuestra que si bien el uso de técnicas broncoscópicas no genera impacto en la mortalidad, tiempo de estancia en UCI, duración de la ventilación mecánica o estancia hospitalaria su uso permite ajustar el espectro antibiótico y lograr deescalar más rápido el tratamiento $(12,13$, 19). Aunque las primeras experiencias con deescalamiento son limitadas, los datos disponibles sugieren que los resultados podrían mejorar con su uso. Estos resultados incluyen menor uso de antibióticos, menor número de episodios secundarios de neumonía, menor resistencia antibiótica, menor duración y eventualmente reducción de la mortalidad (24-26).

Datos recientes sugieren beneficio, con disminución de la mortalidad asociada a la decisión de deescalar la terapia antibiótica en pacientes en shock séptico y sepsis severa (32). La publicación presentada por Joung et al. en 2011 (33), reporta inclusión de pacientes con neumonía nosocomial en quienes se practicó aislamiento microbiológico mediante toma de lavado broncoalveolar o aspirado traqueal. Se observó que aquellos en quienes se logró deescalamiento tuvieron una tasa de mortalidad significativamente más baja en comparación con los pacientes del grupo de no deescalamiento a los 14 (2,3 vs. $10,8 \%$, respectivamente; $\mathrm{p}=0,08)$ y 30 días $(2,3$ vs. $14 \%$, respectivamente; $\mathrm{p}=0,03$ ) de seguimiento.

\section{Cultivos cuantitativos vs. cualitativos}

En 2014 se publicó el metaanálisis de Breton al (34), en el cual se analiza la utilidad de los cultivos cuantitativos vs. Los cualitativos. La mortalidad por todas las causas acumulada fue del 25,4\% (159/626) en el grupo de aislamientos cualitativos y el $23,1 \%$ $(142 / 614)$ en el cuantitativos. No hubo diferencias estadísticamente significativas entre el uso de cultivos cuantitativos vs. cualitativos (RR 0,91; 95\% IC $0,75$ a 1,11$)$.

En la actualidad, sin embargo, se requieren más datos sobre esta estrategia y la utilidad de los cultivos cuantitativos o cualitativos para su implementación.

Conocer el comportamiento epidemiológico de las instituciones, teniendo clara la prevalencia de los principales microorganismos causales de las infecciones respiratorias, puede llegar a ser la mejor oportunidad para lograr iniciar de forma empírica el tratamiento apropiado, para lo cual es necesario contar con aislamientos microbiológicos con puntos de corte confiables (cultivos cuantitativos) que permitan distinguir entre gérmenes producto de colonización vs. gérmenes etiológicos o causales. 
Tabla 1. Informe GRUVECO año 2010 (20).

\begin{tabular}{|lcc|}
\hline & Frecuencia & Porcentaje \\
\hline Aspirado endotraqueal cuantitativo & 27 & 69,2 \\
\hline Aspirado endotraqueal cualitativo & 5 & 12,8 \\
\hline Lavado broncoalveolar broncoscópico & 5 & 12,8 \\
Lavado broncoalveolar no broncoscópico & 2 & 5,1 \\
Total & $\mathbf{3 9}$ & $\mathbf{1 0 0 , 0}$ \\
\hline
\end{tabular}

Para nuestra población se sabe, a través de los datos aportados por GRUVECO (20), que la técnica más utilizada para el diagnóstico de neumonía asociada a la ventilación es el aspirado traqueal $(69,2 \%)$, pese e ello, el número de cultivos para el estudio de la NAVM continúa siendo bajo (tabla 1).

Diferentes análisis (27) de la información existente demuestran que el uso del estudio microbiológico no aumenta la sensibilidad en el diagnóstico de NAVM. La aproximación en el diagnóstico y el tratamiento de pacientes con NAVM, incluye el inicio temprano de la terapia antibiótica y la posterior rectificación de acuerdo con la respuesta clínica y el resultado de cultivos.

\section{Conclusiones}

1. La evidencia existente en los diferentes ensayos clínicos y metaanálisis (23) no sugiere beneficio en términos de mortalidad, días de ventilación mecánica o estancia en UCI al comparar técnicas invasivas frente a no invasivas.

2. El inicio temprano y apropiado de antibiótico, independiente del método diagnóstico utilizado, es la única medida de intervención que ha demostrado mejores resultados en términos de supervivencia de los pacientes con $\operatorname{NAVM}(6,17,18)$.

3. Lograr deescalar la terapia antibiótica en infecciones severas, shock séptico y neumonía nosocomial se puede asociar con la política de uso apropiado de antibióticos y disminución de la mortalidad.

4. El beneficio de los métodos de aislamiento microbiológico y la realización de cultivos cuantitativos se asocian con la evaluación en el uso apropiado del tratamiento y la disminución del tratamiento antibiótico innecesario.

5. Los cultivos cuantitativos son la fuente de información para el conocimiento de los gérmenes causales más frecuentes.

6. Se recomienda realizar aislamiento microbiológico en pacientes con sospecha NAVM mediante la mejor técnica disponible en el sitio de práctica clínica.

\section{Conflictos de interés}

Los autores declaran no tener conflictos de interés.

\section{Bibliografía}

1. Wunderink RG, Woldenberg LS, Zeiss J, Day CM, Ciemins $\mathrm{J}$, Lacher DA. The radiologic diagnosis of autopsy-proven ventilator-associated pneumonia. Chest. 1992;101(2):458.

2. Andrews CP, Coalson JJ, Smith JD, Johanson WG Jr. Diagnosis of nosocomial bacterial pneumonia in acute diffuse lung injury. Chest. 1981;80(3):254.

3. American Thoracic Society. Infectious Diseases Society of America Guidelines for the management of adults with hospital-acquired, ventilator-associated, and healthcare-associated pneumonia. Am J Respir Crit Care Med. 2005;171(4):388.

4. Rubin SA, Winer-Muram HT, Ellis JV. Diagnostic imaging of pneumonia and its complications in the critically ill patient. Clin Chest Med. 1995;16(1):45.

5. Kollef MH. What is ventilator associated pneumonia and why is it important? Respiratoy Care. 2005;50:714-21.

6. Iregui M, Ward S, Sherman G, Fraser VJ, Kollef MH. Clinical importance of delays in the initiation of appropriate antibiotic treatment for ventilator-associated pneumonia. CHEST. 2002;122(1):262-8. 
7. Luna CM, Aruj P, Niederman MS, Garzón J, Grupo Argentino de Estudio de la Neumonía Asociada al Respirador (GANAR) group Appropriateness and delay to initiate therapy in ventilator-associated pneumonia. Eur Respir J. 2006;27:158-64.

8. Kollef MH, Fraser VJ. Antibiotic resistance in the intensive care unit. Ann Intern Med. 2001;134(4):298-314.

9. Torres A, El-Ebiary M. Bronchoscopic BAL in the diagnosis of ventilator-associated pneumonia. Chest. 2000;117:198S$202 \mathrm{~S}$.

10. de Jaeger A, Litalien C, Lacroix J. Protected specimen brush or bronchoalveolar lavage to diagnose bacterial nosocomial pneumonia in ventilated adults: A meta-analysis. Critical Care Medicine. 1999;27(11):2548-60.

11. Lim W, Baudouin S, George R, Hill A, Jamieson C, Jeune I, et al. British Thoracic Society guidelines for the management of community acquired pneumonia in adults: update 2009. Thorax. 2009; 64 (Suppl III):iii1-iii55.

12. Fagon JY, Chastre J, Wolff M, Gervais C, Parer-Aubas S, Stéphan F, Invasive and noninvasive strategies for management of suspected ventilator-associated pneumonia. A randomized trial. Ann Intern Med. 2000;132(8):621-30.

13. Sanchez-Nieto JM, Torres A, Garcia-Cordoba F, El-Ebiary M, Carrillo A, Ruiz J, Nuñez ML, Niederman M. Impact of invasive and noninvasive quantitative culture sampling on outcome of ventilator-associated pneumonia: a pilot study. Am J Respir Crit Care Med. 1998;157:371-6.

14. Shorr AF, Sherner JH, Jackson WL, Kollef MH. Invasive approaches to the diagnosis of ventilator-associated pneumonia: a meta-analysis. Crit Care Med. 2005;33(1):46-53.

15. Ruiz M, Torres A, Ewig S. Noninvasive Versus Invasive Microbial Investigation in Ventilator-associated Pneumonia: Evaluation of Outcome. Am J Respir Crit Care Med. 2000;162:119-25.

16. Solé Violán J, Fernández JA, Benítez AB, Cardeñosa Cendrero JA, Rodríguez de Castro F. Crit Care Med. 2000 Aug;28(8):2737-41. Impact of quantitative invasive diagnostic techniques in the management and outcome of mechanically ventilated patients with suspected pneumonia. Crit Care Med. 2000;28(8):2737-41.

17. Alvarez-Lerma F. Modification of empiric antibiotic treatment in patients with pneumonia acquired in the intensive care unit. Int Care Med. 1996;22(5):387-94.

18. Luna CM, Vujacich P, Niederman MS, Vay C, Gherardi C, Matera J, Jolly EC. Impact of BAL data on the therapy and outcome of ventilator-associated pneumonia. Chest. 1997; 111(3):676-85.

19. Canadian Critical Care Trials Group. A randomized trial of diagnostic techniques for ventilator-associated pneumonia. N Engl J Med. 2006; 355(25):2619-30.

20. Ortiz G, Garay M, Fonseca N, Molina F, Lara A, Dueñas C, Epidemiología de la neumonía asociada a ventilador en 39 unidades de cuidados intensivos de Colombia (2007-2009). Informe año 2010. Act Colomb Cuidado Int. 2011;11(1):12-9.
21. Hoffken G, Niederman MS. Nosocomial pneumonia: the importance of a de-escalating strategy for antibiotic treatment of pneumonia in the ICU. Chest. 2002;122:2183-96.

22. O'Horo J, Thompson D, Safdar N. Is the Gram Stain Useful in the Microbiologic Diagnosis of VAP? A Meta-analysis. Clin Infect Dis. 2012;55(4):551-61.

23. Berton DC, Kalil AC, Teixeira PJ. Quantitative versus qualitative cultures of respiratory secretions for clinical outcomes in patients with ventilator-associated pneumonia. Cochrane Database Syst Rev. 2012;1:CD006482.

24. Soo Hoo GW, Wen E, Nguyen TV, Goetz MD. Impact of clinical guidelines in the management of severe hospital-acquired pneumonia. Chest. 2005;128:2778-87.

25. Rello J, Vidaur L, Sandiumenge A, et al. De-escalation therapy in ventilator associated pneumonia. Crit Care Med. 2004;32:2183-90.

26. Kollef MH, Morrow LE, Niederman MS, et al. Clinical characteristics and treatment patterns among patients with ventilator-associated pneumonia. Chest. 2006;129:1210-8.

27. Rea-Neto A, Tuche F, Brunkhorst F, Ranieri M, Reinhart K, Sakr Y. Diagnosis of ventilator-associated pneumonia: a systematic review of the literature. Crit Care. 2008;12:R56.

28. Chastre J, Fagon JY, Bornet-Lecso M, et al. Evaluation of bronchoscopic techniques for the diagnosis of nosocomial pneumonia. Am J Respir Crit Care Med. 1995;152:231-40.

29. Marquette $\mathrm{CH}$, Georges H, Wallet F, et al. Diagnostic efficiency of endotracheal aspirates with quantitative bacterial cultures in intubated patients with suspected pneumonia. Comparison with the protected specimen brush. Am Rev Respir Dis. 1993;148:138-44.

30. Fagon JY, Chastre J, Domart Y et al. Nosocomial pneumonia in patients receiving continuous mechanical ventilation. Prospective analysis of 52 episodes with use of a protected specimen brush and quantitative culture techniques. Am Rev Respir Dis. 1989;139:877-84.

31. Torres A, Puig de la BJ, Xaubet A, et al. Diagnostic value of quantitative cultures of bronchoalveolar lavage and telescoping plugged catheters in mechanically ventilated patients with bacterial pneumonia. Am Rev Respir Dis. 1989;140:306-10.

32. Garnacho-Montero J, Gutiérrez-Pizarraya A, EscorescaOrtega A, Corcia-Palomo Y, Fernández-Delgado E, HerreraMelero I, et al. De-escalation of empirical therapy is associated with lower mortality in patients with severe sepsis and septic shock. Int Care Med. 2014;40(1):32-40.

33. Joung MK, Lee JA, Moon SY, Cheong HS, Joo EJ, Ha YE, et al. Impact of de-escalation therapy on clinical outcomes for intensive care unit-acquired pneumonia. Crit Care. 2011;15(2):R79.

34. Berton D, Kalil AC, Teixeira P. Quantitative versus qualitative cultures of respiratory secretions in patients with ventilator-associated pneumonia. Cochrane Database Syst Rev. 2014;30(10):CD006482. 